\title{
Trace Conditioning and the Hippocampus: The Importance of Contiguity
}

\author{
Debra A. Bangasser, David E. Waxler, Jessica Santollo, and Tracey J. Shors \\ Department of Psychology and Center for Collaborative Neuroscience, Rutgers University, Piscataway, New Jersey 08854
}

Trace conditioning, a form of classical conditioning in which the presentation of the conditioned stimulus (CS) and the unconditioned stimulus (US) is separated in time by an interstimulus interval, requires an intact hippocampus. In contrast, classical conditioning procedures in which the CS and US are not separated by an interstimulus interval (i.e., delay conditioning procedures) typically do not (Solomon et al., 1986). However, why trace conditioning is dependent on the hippocampus is unknown. Several theories suggest that it is specifically the discontiguity between the CS and US in trace conditioning that critically engages the hippocampus. However, there are other explanations that do not depend on discontiguity. To determine whether the lack of contiguity renders trace conditioning hippocampal dependent, we designed a "contiguous trace conditioning" (CTC) paradigm in which CS-US contiguity is restored by representing the CS simultaneously with the US. Although rats with excitotoxic lesions of the hippocampus could not learn a standard trace fear-conditioning paradigm, lesioned rats trained on CTC showed significant conditioning, at levels similar to those with sham surgeries. Importantly, lesioned rats trained solely with simultaneous CS-US presentations did not demonstrate conditioning. Together, these data suggest that rats with hippocampal lesions can form a memory of a trace CS-US association when contiguity is restored. Therefore, the dependence of traditional trace paradigms on the hippocampus can be attributed to the absence of temporal contiguity.

Key words: associative learning; fear conditioning; learning; memory; trace conditioning; time

\section{Introduction}

The defining difference between delay and trace classical conditioning is simple: in delay conditioning, the unconditioned stimulus (US) immediately follows or coterminates with the conditioned stimulus (CS), whereas in trace conditioning, the CS and US are separated in time by a "trace" interval. Although simple, this difference can have profound effects on learning. First, subjects usually require more trials to acquire trace conditioning than delay (Pavlov, 1927; Beylin et al., 2001). Second, trace conditioning requires an intact hippocampus, whereas delay conditioning does not (Solomon et al., 1986; McEchron et al., 1998). In one study, lesioned rats showed no evidence of learning trace eyeblink conditioning even after 1000 training trials (Beylin et al., 2001). However, why trace conditioning is dependent on the hippocampus is unknown.

Because the presence of a trace interval renders the CS and US temporally discontiguous, it has been proposed that the hippocampus is used to overcome stimulus discontiguity (Wallenstein et al., 1998). For example, some theories hold that the hippocampus is used to maintain a memory trace of the CS so that it can be associated with the US later in time. Another possibility is that the training context, which is contiguous with both the CS

Received April 24, 2006; revised June 12, 2006; accepted June 16, 2006.

This work was supported by National Institute of Mental Health Grants MH59970 and MH59740 and National Science Foundation Grant IOB-0444364. We thank Drs. C. R. Gallislet and L. Matzel for comments.

Correspondence should be addressed to Tracey J. Shors, Department of Psychology, Rutgers University, 152 Frelinghuysen Road, Piscataway, NJ 08854. E-mail: shors@rutgers.edu.

DOI:10.1523/JNEUROSCI.1742-06.2006

Copyright (C) 2006 Society for Neuroscience $\quad$ 0270-6474/06/268702-05\$15.00/0 and US, acts as a bridge between the stimuli (Quinn et al., 2002). This would account for hippocampal involvement in trace conditioning because rats with hippocampal lesions show an impaired ability to use contextual stimuli (Kim and Fanselow, 1992; Phillips and LeDoux, 1992; Anagnostaras et al., 2001).

Other theories do not rely on CS-US discontiguity. One theory suggests that the hippocampus is necessary for timing the conditioned responses in trace eyeblink conditioning (Solomon et al., 1986; James et al., 1987). Another theory suggests that trace conditioning may be more difficult, and possibly hippocampal dependent, because subjects must discriminate the trace interval from the intertrial interval (Mowrer and Lamoreaux, 1951; Bolles et al., 1978; Kaplan and Hearst, 1982). Finally, some research suggests that trace conditioning depends on a hippocampal form of awareness (Clark and Squire, 1998, 2004).

Because several theories rely on stimulus discontiguity and several do not, determining the importance of contiguity could reduce the number of viable explanations. To this end, we devised a "contiguous trace conditioning" (CTC) paradigm that is similar to the standard trace fear-conditioning paradigm (i.e., a white-noise CS is separated from a footshock US by a $30 \mathrm{~s}$ trace interval). However in CTC, instead of the US being presented alone, it is presented simultaneously with a second CS (see Fig. $2 A$ ). Because simultaneous conditioning results in little to no conditioned responding (Matzel et al., 1988), the addition of a simultaneous component to the trace arrangement adds CS-US contiguity, without increasing the predictive relationship between stimuli. If stimulus discontiguity is the reason why trace conditioning is hippocampal dependent, animals with hippocampal lesions should be able to learn the CS-US association 
after training with the CTC procedure. However, if trace conditioning requires the hippocampus for other reasons, lesioned rats should be unable to acquire the conditioned response during training with the CTC procedure.

\section{Materials and Methods}

Subjects. Forty-two male Sprague Dawley rats (60-90 d of age; 350-500 g) were bred from Harlan stock. They were housed singly, provided with ad libitum access to food and water, and maintained on $12 \mathrm{~h}$ light/dark cycles.

Surgery. Rats were anesthetized with sodium pentobarbital $(50 \mathrm{mg} / \mathrm{kg}$, i.p.) and atropine $(0.04 \mathrm{mg} / \mathrm{kg}$, i.p.) to reduce salivary secretions. Boosters of each were given as needed. After securing the rat in the stereotaxic instrument, the tissue and skull over the hippocampus were removed. A syringe (10 $\mu$; Hamilton, Reno, NV) containing NMDA $(20 \mathrm{mg} / \mathrm{ml}$ in PBS) was lowered to 12 sites throughout the hippocampus [anteroposterior $(\mathrm{AP})-2.5 \mathrm{~mm}$, mediolateral $(\mathrm{ML}) \pm 1.6$, dorsoventral $(\mathrm{DV})-3.8$ $\mathrm{mm}$; AP $-4.2 \mathrm{~mm}$, ML $\pm 2.6, \mathrm{DV}-3.1 \mathrm{~mm}$; AP $-5.3 \mathrm{~mm}$, ML $\pm 5.0 \mathrm{~mm}, \mathrm{DV}-5.9 \mathrm{~mm} ; \mathrm{AP}-5.3 \mathrm{~mm}, \mathrm{ML} \pm 4.2 \mathrm{~mm}, \mathrm{DV}-3.4 \mathrm{~mm}$; AP $-5.8 \mathrm{~mm}, \mathrm{ML} \pm 4.6 \mathrm{~mm}, \mathrm{DV}-6.1 \mathrm{~mm} ; \mathrm{AP}-6.0 \mathrm{~mm}, \mathrm{ML} \pm 5.6 \mathrm{~mm}, \mathrm{DV}$ $-4.1 \mathrm{~mm}$; with AP coordinates measured relative to bregma, ML coordinates measured from the midline, and DV coordinates measured from the surface of the brain]. After waiting $1 \mathrm{~min}$ at each site for the tissue around the syringe to settle, NMDA $(0.35 \mu \mathrm{l})$ was infused via a microinfuser pump (at a rate of $0.5 \mu \mathrm{l} / \mathrm{min}$; Stoelting, Wood Dale, IL). After each infusion, the syringe was left in place for 2 min to allow for diffusion of the compound. Once the infusions were complete, the hole in the skull was covered with bone wax, and the incision was closed with wound clips. For sham-operated control animals, surgical procedures remained similar, except that the syringe was lowered only through the overlying cortex (DV, $-2.5 \mathrm{~mm})$ at all 12 injection sites and no compound was infused. Animals were allowed at least 1 week to recover before training.

Conditioning apparatus. Four conditioning boxes (SD Instruments, San Diego, CA) were used. Inside of each sound-attenuating outer chamber was an inner chamber $(25 \times 21 \times 18.5 \mathrm{~cm})$ with plastic walls, a removable plastic lid, and a floor grid. A computer program (SD Instruments) controlled a white-noise generator attached to a speaker that was used to administer a $70 \mathrm{~dB}$ white noise (CS) and a shock generator attached to the floor grid that was used to administer a scrambled, $0.5 \mathrm{~mA}$ shock (US). The chambers could be made distinct for training and testing phases by changing several contextual cues, including wall patterns (white vs $7.6 \mathrm{~cm}$ alternating black and white vertical stripes), odorants (clean air, peppermint, or coconut), and flooring (stainless steel rods vs Plexiglas floor cover). A photobeam activity system measured horizontal and vertical movement, which were used to quantify the degree of fear conditioning.

Fear-conditioning procedure. Fear conditioning was administered over $3 \mathrm{~d}$ and consisted of a pre-exposure phase, a training phase, and a testing phase. During the pre-exposure phase, subjects were acclimated to the conditioning chambers for $10 \mathrm{~min}$, and the white-noise stimulus was presented twice for $15 \mathrm{~s}$. Twenty-four hours later, subjects were trained on one of four conditioning procedures: trace, CTC, simultaneous, or delay (Fig. 2 A). Trace conditioning consisted of a $15 \mathrm{~s}$ CS presentation, followed by a $30 \mathrm{~s}$ trace interval, and then a $2 \mathrm{~s}$ US presentation. CTC consisted of a $15 \mathrm{~s}$ CS presentation, followed by a $30 \mathrm{~s}$ trace interval, and then a $2 \mathrm{~s}$ simultaneous CS-US presentation. Simultaneous conditioning consisted of a $2 \mathrm{~s}$ CS-US presentation. Delay conditioning consisted of a $47 \mathrm{~s}$ CS that overlapped and coterminated with a $2 \mathrm{~s}$ US. All of the training procedures consisted of five training trials with an intertrial interval of 5 min. Twenty-four hours later, animals were presented with one CS alone test trial in a chamber that was contextually distinct from the chamber in which they were trained.

For testing, the amount of conditioned responding was quantified by measuring the percentage of change in movement in response to a CS presentation. This was accomplished by comparing the activity in response to the test $\mathrm{CS}$ to a baseline period, which was measured during the 30 s period before the CS onset. Rats with $<10$ beam breaks during this baseline period were eliminated from the study, because their baseline
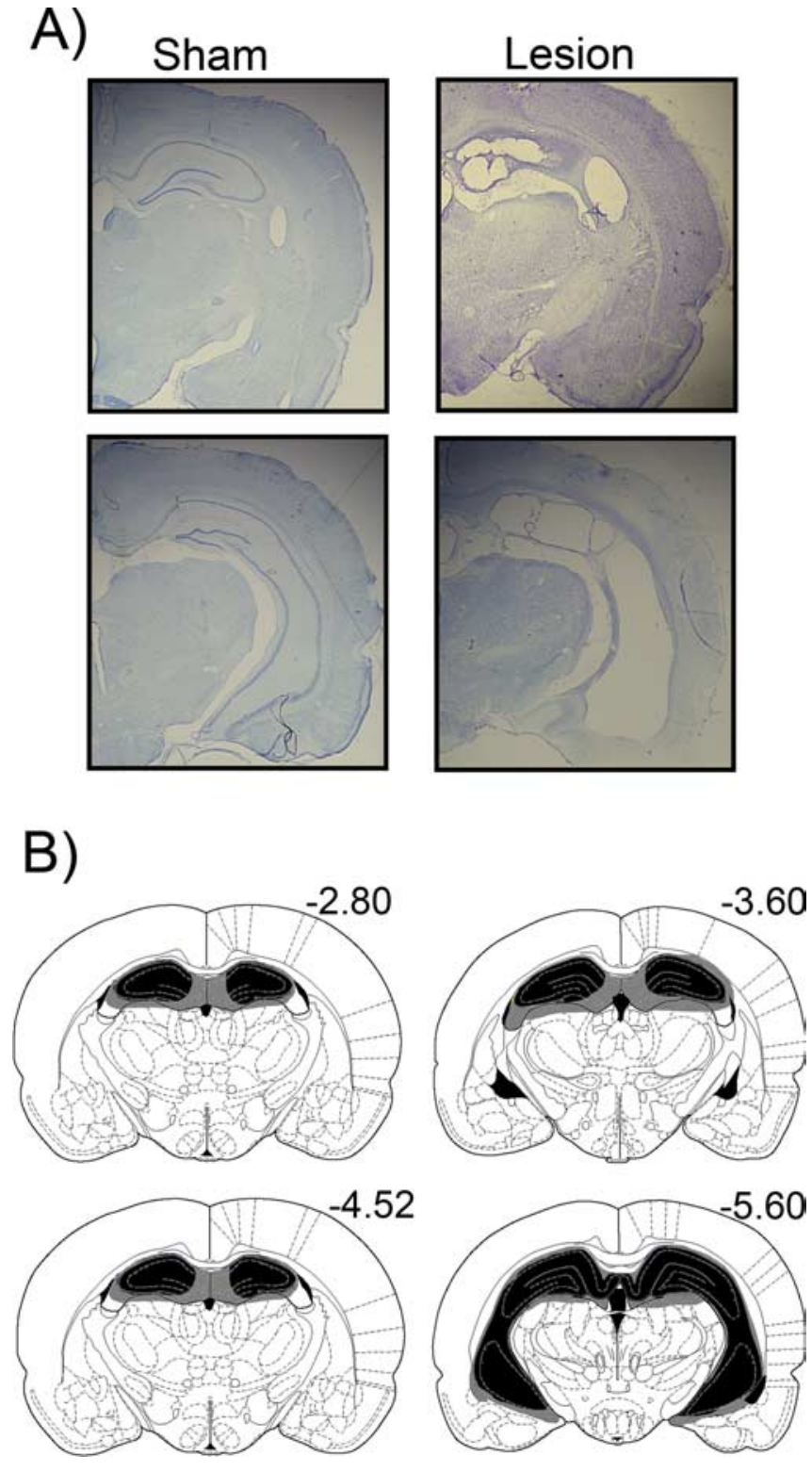

Figure 1. Subjects in the experimental group were given excitotoxic lesions of the complete hippocampus. $\boldsymbol{A}$, Representative photomicrograph of tissue from a sham-operated control (left column) and hippocampal-lesioned rat (right column). Substantial cell loss was observed throughout the dorsal and ventral hippocampus. $\boldsymbol{B}$, The drawing depicts the largest (in gray) and smallest (in black) lesion selected from all subjects included in the study, shown here according to the atlas of Paxinos and Watson (1997).

activity was deemed insufficient to reliably detect any fear-induced decrease. The test stimulus for subjects trained on the trace, CTC, and simultaneous procedures consisted of one 15 s CS presentation. Movements during the $30 \mathrm{~s}$ period after the CS presentation (when the trace interval had occurred during training) were compared with the pre-CS baseline. The testing procedure for delay conditioning consisted of one $47 \mathrm{~s}$ CS presentation. During testing for delay conditioning, movements during the equivalent period (i.e., the last $30 \mathrm{~s}$ of the CS presentation) were compared with baseline activity.

Histology. After testing, rats were deeply anesthetized and perfused transcardially with $10 \%$ formalin. Brains were extracted and postfixed in a $10 \%$ formalin solution with $30 \%$ sucrose solution added before cutting. Coronal sections $(50 \mu \mathrm{m})$ containing target regions were cut on a cryostat, and every fifth section was mounted onto gelled slides. Finally, tissue was stained with cresyl violet. A rater who was blind to the behavioral 
A)
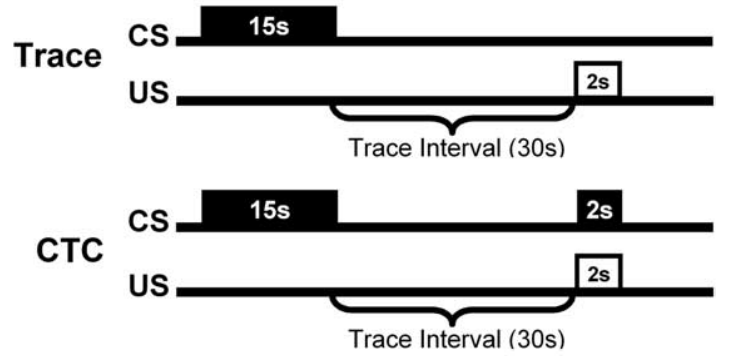

Simultaneous
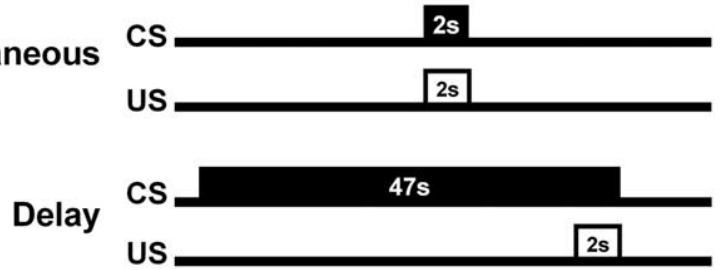

\section{B)}

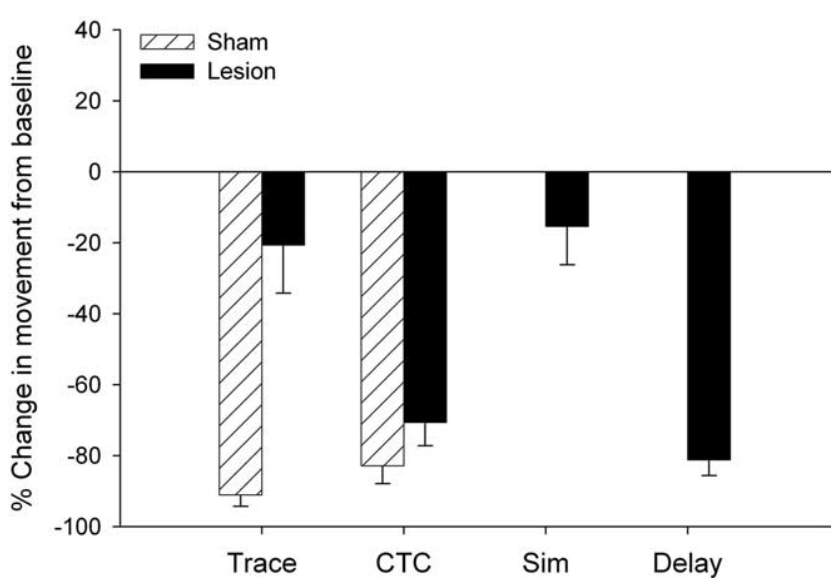

Figure 2. Hippocampal-lesioned subjects were able to form a memory of the trace relationship when trained with the CTC procedure but not when trained with trace conditioning. $A$, This schematic represents the temporal relationship between the $C S$ and US for a single trial for the following four conditioning procedures: trace, CTC, simultaneous, and delay conditioning. $\boldsymbol{B}$ The percentage of change in movement from the baseline was calculated to quantify conditioned fear to the CS during testing. Rats with hippocampal lesions that were trained with delay conditioning showed conditioned responding, which indicates that the lesions did not disrupt conditioning on tasks that do not require the hippocampus. Unlike sham-operated controls, rats with hippocampal lesions trained with trace conditioning did not show conditioned responding. However, rats with hippocampal lesions trained with the CTC procedure showed significant responding at levels similar to their sham-operated counterparts. Because lesioned subjects trained with simultaneous conditioning alone did not emit conditioned responses, the responding expressed by subjects in the CTC lesion group cannot be attributed to the simultaneous component of the training procedure. Together, these results suggest that rats with lesions of the hippocampus can form a memory of the CS-US association if they are trained with CTC, in which contiguity is restored. Error bars indicate SEM.

data made qualitative lesion assessments. Only subjects with bilateral damage to the dorsal and ventral hippocampus were included in the study (Fig. 1A). These criteria resulted in the following number of animals in each condition: trace sham, $n=7$; trace lesion, $n=7$; CTC sham, $n=7$; CTC lesion, $n=8$; simultaneous lesion, $n=6$; delay lesion, $n=7$. Representative lesions are shown in Figure $1 B$.

\section{Results}

Hippocampal lesions prevented trace but not delay fear conditioning

As described in Materials and Methods, the amount of conditioned responding during testing was quantified by measuring the percentage of change in movement in response to a CS presentation. To make sure that the hippocampal lesions impaired trace conditioning, without disrupting delay conditioning, data from rats with hippocampal lesions trained on these procedures were analyzed. A one-sample $t$ test revealed that lesioned rats trained on trace conditioning showed no significant conditioned responding to the CS during testing $\left(\mu=0 ; t_{(6)}=-1.53 ; p=\right.$ 0.176 ) (Fig. $2 B$ ). In contrast, the same analysis revealed that lesioned rats trained on delay conditioning showed significant responding to the CS during testing $\left(\mu=0 ; t_{(5)}=-18.04 ; p<\right.$ $0.001)$. Furthermore, an independent-samples $t$ test revealed that there was a significant difference between the two groups $\left(t_{(11)}=\right.$ 3.98; $p=0.002$ ); animals with hippocampal lesions that were trained on delay conditioning showed more conditioned responding than those trained on trace conditioning. As was expected, these analyses indicate that hippocampal lesions prevented trace but not delay fear conditioning.

\section{Hippocampal lesions did not prevent CTC}

Conditioned responding from the animals that were trained on the traditional trace procedure was compared with responding from animals that were trained on the CTC procedure using a $2 \times$ 2 ANOVA with surgery (lesion or sham) and conditioning procedure (CTC or trace) as the factors. The analysis revealed an interaction between surgery and training procedure $\left(F_{(1,23)}=\right.$ $11.15 ; p=0.003)$. Newman-Keuls post hoc tests further revealed that sham-operated rats trained on trace conditioning showed more conditioned responding than did the lesioned rats trained on trace conditioning $(p<0.001)$. Lesioned rats trained on the CTC procedure showed more conditioned responding than did the lesioned rats trained on the standard trace procedure $(p<$ 0.001 ), and their responding was not different from those in sham-operated controls $(p>0.05)$.

Rats trained on simultaneous conditioning alone showed no significant responding during testing

Because it was possible that the simultaneous component of the CTC procedure caused the change in conditioned responding on its own, we compared conditioning from lesioned animals that were trained on simultaneous conditioning to lesioned animals that were trained on the CTC procedure. An independentsamples $t$ test indicated that the animals trained on CTC showed more conditioned responding than did the animals trained with the simultaneous conditioning $\left(t_{(11)}=-5.41 ; p=0.03\right)$. Furthermore, a one-sample $t$ test revealed that the simultaneous group showed no significant conditioned responding during testing $\left(\mu=0 ; t_{(6)}=-1.84 ; p=0.116\right)$. These results indicate that simultaneous conditioning alone did not lead to conditioned responding.

\section{Discussion}

The present study was designed to determine whether it is specifically the discontiguity between the CS and the US, and not other factors, that renders trace conditioning hippocampal dependent. To test this hypothesis, we designed the CTC procedure, which restored CS-US contiguity (by re-presenting the CS again simultaneously with the US after the trace interval) but does not increase the predictive relationship between the CS and US. As 
expected, rats with hippocampal lesions trained with trace conditioning did not emit conditioned responses to the CS, indicating that, unlike sham-operated controls, they could not form a memory of the trace relationship. In contrast, rats with hippocampal lesions that were trained with the CTC procedure showed significant responding to the CS during testing, at levels similar to rats with sham surgeries. Their responding could not be attributed to the simultaneous component of CTC because lesioned rats trained with the simultaneous stimuli alone did not demonstrate conditioned responding. Together, these results indicate that animals without a functional hippocampus can form a memory of a trace CS-US association when contiguity is restored.

\section{Revaluating theories that do not rely on CS-US discontiguity} Our results suggest that theories that do not rely on CS-US discontiguity to explain the role of the hippocampus in trace conditioning, may not be supported. One such theory suggests that the hippocampus is used to time conditioned responses during trace conditioning (Solomon et al., 1986; James et al., 1987). However, in the present study, rats could emit conditioned responses when trained on CTC, which has the same trace interval and CS duration as our trace procedure; therefore, the timing theory is not supported. Another theory suggests that the hippocampus is used to discriminate the trace interval from the intertrial interval. However, this theory is probably not correct because the addition of the simultaneous component to the CTC procedure did nothing to explicitly disambiguate these intervals.

Some theories do not make explicit predictions with regard to the role of contiguity in trace conditioning. In most cases, the results of the current study neither support nor contradict these theories directly, but do suggest a need to integrate stimulus discontiguity into their models. For example, it has been proposed that trace memories represent a form of declarative memory that requires awareness on the part of the subject, and the hippocampus is therefore involved (Clark and Squire, 1998, 2004). If this theory is correct, then it is conceivable that it is specifically the discontiguous arrangement of the stimuli in trace conditioning that engages processes involved in the formation of declarative memories.

Of course, when discussing theoretical models of trace conditioning, it is important not to generalize too broadly between eyeblink and fear-conditioning procedures. There are many differences between these two types of trace conditioning tasks, including distinct neural substrates. However, despite the paradigmatic and anatomical differences between trace eyeblink and trace fear conditioning, the role of the hippocampus seems to be similar. Therefore, it is likely that animals with hippocampal lesions could acquire a CTC version of eyeblink conditioning.

\section{How the hippocampus could associate stimuli that are discontiguous in time}

The results of the current study suggest that the hippocampus is involved in overcoming CS-US discontiguity in trace conditioning. Exactly how the hippocampus might do this is still unclear. One possibility is that the hippocampus bridges the gap between the CS and US by creating "artificial" CS-US contiguity within the brain. For example, the CS could be held in a type of Hebbian reverberating circuit until the US is presented (Gormezano et al., 1983). If true, one might expect to find neurons in the hippocampus with behavioral correlates similar to the "delay cells" found in the prefrontal cortex of primates (i.e., the hippocampal neurons might represent the CS by firing throughout the trace interval)
(Fuster and Alexander, 1971). To our knowledge, this type of response has not been demonstrated in the hippocampus. However, some hippocampal cells do demonstrate increased firing, which eventually coincides with the scheduled occurrence of the US (Solomon et al., 1986; McEchron and Disterhoft, 1997; McEchron et al., 2003). Based on these and other data, it has been suggested that the hippocampus indirectly represents the CS through a relay of neuronal activity, which culminates in time at the end of the trace interval (Rodriguez and Levy, 2001). An alternative view of how the hippocampus may overcome stimulus discontiguity suggests that contextual information, which can be associated with both the CS and US, acts like a bridge between the two stimuli (Quinn et al., 2002). This theory is appealing because the hippocampus is thought to be critically involved in contextual conditioning (Kim and Fanselow, 1992; Phillips and LeDoux, 1992; Anagnostaras et al., 2001).

\section{Other possible roles for the hippocampus}

An alternative interpretation of the present results is that the hippocampus is involved in task difficulty. Previously, we manipulated a delay eyeblink conditioning procedure by lengthening the CS (Beylin et al., 2001). Like trace conditioning, this "long delay" procedure was more difficult to acquire than standard delay procedures. Importantly, this long delay procedure was shown to be dependent on an intact hippocampus. One interpretation of these findings is that the hippocampus becomes critically engaged when tasks become more difficult to learn (Beylin et al., 2001; Shors, 2004). Similarly, with respect to the present data, it could be argued that restoring CS-US contiguity in the CTC procedure renders the task easier to learn, and therefore independent of the hippocampus. Unfortunately, we cannot assess this possibility here because the sham-operated control animals trained with trace and CTC responded at ceiling levels. However, even if CTC is easier to learn than the standard trace procedure, it would not change the central conclusion of this study, namely that discontiguity, and not other factors, critically engages the hippocampus during trace conditioning.

\section{Traditional versus modern interpretations of association formation}

The present results suggest that stimulus contiguity plays an important role in conditioning. This idea is consistent with traditional theories of associative learning, which hold that two stimuli presented in close spatial or temporal contiguity will become associated together (Pavlov, 1927). However, several modern theories of associative learning downplay the importance of contiguity, suggesting that it is neither necessary nor sufficient for association formation (Egger and Miller, 1962; Kamin, 1969; Rescorla, 1969, 1988; Rescorla and Wagner, 1972). The charge that contiguity is insufficient to lead to learning is based mainly on simultaneous and blocking procedures, which have CS-US contiguity but which result in little or no conditioned responding. However, careful probing has revealed that associations are formed in these procedures, even if the behavior is silent during typical testing conditions (Dickinson et al., 1976; Matzel et al., 1988). With regard to whether contiguity is a necessary condition for association formation, trace conditioning is often cited as a counter example. However, the current study suggests an important role for contiguity in trace fear conditioning, even if intact animals can still associate discontiguous stimuli. It could be argued, therefore, that the hippocampus has evolved the special role of compensating for discontiguity.

How then do we reconcile modern associative theories (which 
emphasize predictability, information, and timing as key factors for learning) with a role for contiguity (Egger and Miller, 1962; Kamin, 1969; Rescorla, 1969, 1988; Rescorla and Wagner, 1972)? In the current study, rats with hippocampal lesions were able to learn the predictive relationship between stimuli, but only after contiguity was restored. In contrast to the view that the predictive relationship, by itself, leads to association formation, these results may indicate that the predictive relationship can only be learned after the stimuli are first associated by virtue of their contiguity. In this way, contiguity creates a framework on which other more complicated representations are built. Based on this interpretation, one would predict that rats with hippocampal lesions would form a memory of the trace relationship if they were first trained with contiguous stimuli. In fact, Beylin et al. (2001) found that rats with hippocampal lesions were able to form a memory of the trace relationship if they had previously learned to associate the $\mathrm{CS}$ and US during training on delay conditioning. These animals were even able to adjust the timing of their conditioned response to fit the temporal relationship between the CS and US used for trace conditioning. Interestingly, despite his hippocampal damage, H.M. was also able to learn trace conditioning (WoodruffPak, 1993), perhaps because he was first trained with a delay procedure. Again, these data suggest that the predictive relationships in trace conditioning can be acquired without an intact hippocampus, as long as the stimuli have already become associated together. Perhaps the hippocampus is not as devoted to learning predictive relationships as much as it is used to bind stimuli that do not occur together in time or space.

\section{References}

Anagnostaras SG, Gale GD, Fanselow MS (2001) Hippocampus and contextual fear conditioning: recent controversies and advances. Hippocampus $11: 8-17$.

Beylin AV, Gandhi CC, Wood GE, Talk AC, Matzel LD, Shors TJ (2001) The role of the hippocampus in trace conditioning: temporal discontinuity or task difficulty? Neurobiol Learn Mem 76:447-461.

Bolles RC, Collier AC, Bouton ME, Marlin NA (1978) Some tricks for ameliorating the trace-conditioning deficit. Bull Psychon Soc 11:403-406.

Clark RE, Squire LR (1998) Classical conditioning and brain systems: the role of awareness. Science 280:77-81.

Clark RE, Squire LR (2004) The importance of awareness for eyeblink conditioning is conditional: theoretical comment on Bellebaum and Daum. Behav Neurosci 118:1466-1468.

Dickinson A, Hall G, Mackintosh NJ (1976) Surprise and the attenuation of blocking. J Exp Psychol Anim Behav Processes 2:313-322.

Egger MD, Miller NE (1962) Secondary reinforcement in rats as a function of information value and reliability of the stimulus. J Exp Psychol 64:97-104.

Fuster JM, Alexander GE (1971) Neuron activity related to short-term memory. Science 173:652-654.

Gormezano I, Kehoe EJ, Marshall BS (1983) Twenty years of classical conditioning with the rabbit. Prog Psychobiol Physiol Psychol 10:197-275.
James GO, Hardiman MJ, Yeo CH (1987) Hippocampal lesions and trace conditioning in the rabbit. Behav Brain Res 23:109-116.

Kamin LJ (1969) Predictability, surprise, attention, and conditioning. In: Punishment and aversive behavior (Campbell B, Church RM, eds), pp 279-296. New York: Appleton-Century-Crofts, Educational Division, Meredith.

Kaplan PS, Hearst E (1982) Bridging temporal gaps between CS and US in autoshaping: insertion of other stimuli before, during, and after CS. J Exp Psychol Anim Behav Processes 8:187-203.

Kim JJ, Fanselow MS (1992) Modality-specific retrograde amnesia of fear. Science 256:675-677.

Matzel LD, Held FP, Miller RR (1988) Information and expression of simultaneous and backward associations: implications for contiguity theory. Learn Motiv 19:317-344.

McEchron MD, Disterhoft JF (1997) Sequence of single neuron changes in CA1 hippocampus of rabbits during acquisition of trace eyeblink conditioned responses. J Neurophysiol 78:1030-1044.

McEchron MD, Bouwmeester H, Tseng W, Weiss C, Disterhoft JF (1998) Hippocampectomy disrupts auditory trace fear conditioning and contextual fear conditioning in the rat. Hippocampus 8:638-646.

McEchron MD, Tseng W, Disterhoft JF (2003) Single neurons in CA1 hippocampus encode trace interval duration during trace heart rate (fear) conditioning in rabbit. J Neurosci 23:1535-1547.

Mowrer OH, Lamoreaux RR (1951) Conditioning and conditionality (discrimination). Psychol Rev 58:196-212.

Pavlov IP (1927) Conditioned reflexes. London: Oxford UP.

Paxinos G, Watson C (1997) The rat brain in stereotoxic coordinates, Ed 3. San Diego: Academic.

Phillips RG, LeDoux JE (1992) Differential contribution of amygdala and hippocampus to cued and contextual fear conditioning. Behav Neurosci 106:274-285.

Quinn JJ, Oommen SS, Morrison GE, Fanselow MS (2002) Post-training excitotoxic lesions of the dorsal hippocampus attenuate forward trace, backward trace, and delay fear conditioning in a temporally specific manner. Hippocampus 12:495-504.

Rescorla RA (1969) Information variables in Pavlovian conditioning. In: The psychology of learning and motivation (Bower G, ed). Oxford: Academic.

Rescorla RA (1988) Pavlovian conditioning: it's not what you think it is. Am Psychol 43:151-160.

Rescorla RA, Wagner AR (1972) A theory of Pavlovian conditioning: variations in the effectiveness of reinforcement and nonreinforcement. In: Classical conditioning II: current research and theory (Black AH, Prokasy WF, eds), pp 64-99. New York: Appleton-Century-Crofts.

Rodriguez P, Levy WB (2001) A model of hippocampal activity in trace conditioning: where's the trace? Behav Neurosci 115:1224-1238.

Shors TJ (2004) Memory traces of trace memories: neurogenesis, synaptogenesis and awareness [review]. Trends Neurosci 27:250-256.

Solomon PR, Vander Schaaf ER, Thompson RF, Weisz DJ (1986) Hippocampus and trace conditioning of the rabbit's classically conditioned nictitating membrane response. Behav Neurosci 100:729-744.

Wallenstein GV, Eichenbaum H, Hasselmo ME (1998) The hippocampus as an associator of discontiguous events [review]. Trends Neurosci 21:317-323.

Woodruff-Pak DS (1993) Eyeblink classical conditioning in H.M.: delay and trace paradigms. Behav Neurosci 107:911-925. 